
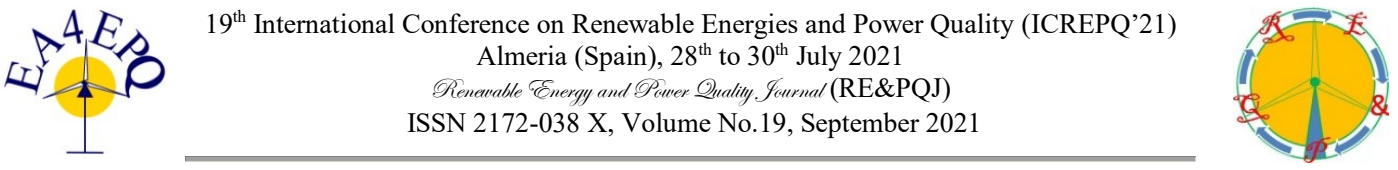

\title{
Neural Network Control of Green Energy Vehicles with Blended Braking Systems
}

\author{
V. Vodovozov ${ }^{1}$, E. Petlenkov ${ }^{2}$, A. Aksjonov ${ }^{1}$ and Z. Raud ${ }^{1}$ \\ ${ }^{1}$ Department of Electrical Power Engineering and Mechatronics \\ ${ }^{2}$ Department of Computer Systems \\ Tallinn University of Technology \\ Ehitajate tee 5, 19086, Tallinn (Estonia) \\ Phone number: +372 53235223, e-mail: valery.vodovozov@gmail.com
}

\begin{abstract}
A neural network-based control system is offered, which ensures high quality blended braking of the green energy vehicles in both the intensive and the gradual deceleration scenarios, with energy recovery at the changing road pavement. In this study, a neural network controller provides the torque gradient control without a tire model resulting in returning maximal energy to the hybrid energy source during the braking process. To meet the conflicting requirements of different braking modes and road surfaces, an allocation algorithm determines how to distribute the driver's torque request between the friction and electrical brakes. Simulation demonstrates effectiveness of the proposed braking system. Model states and inputs are used as a guidance to learn a coupled two-layer neural network capable to capture various dynamic behaviours that could not be included in the simplified physics-based model. An experimental part of the research proves the model and simulation validity.
\end{abstract}

Key words. Electric vehicle, clean energy, neural network, recuperative braking.

\section{Acronyms}

$\begin{array}{ll}\text { ABS } & \text { antilock braking system } \\ \text { EB } & \text { electrical brake } \\ \text { EV } & \text { electric vehicle } \\ \text { FB } & \text { friction brake } \\ \text { HES } & \text { hybrid energy storage } \\ \text { HIL } & \text { hardware-in-the-loop } \\ \mathrm{kJ} & \text { recovered energy } \\ \lambda, \mathrm{L} \% & \text { p.u. and percentage wheel slip, appropriately } \\ \text { MRC } & \text { model reference controller } \\ \text { MSE } & \text { mean squared error } \\ \text { NN } & \text { neural network } \\ \text { NNC } & \text { neural network controller } \\ \text { NNEV } & \text { neural network model of an electric vehicle } \\ r & \text { effective radius of the wheel } \\ \text { REF } & \text { reference } \\ \text { SOC } & \text { state of charge } \\ \text { TA } & \text { torque allocator } \\ \text { T*, T } & \text { actuating and application braking torque, } \\ & \text { appropriately }\end{array}$

$\mathrm{TB}^{*} \quad$ driver's reference

TE*,TE electrical fraction of actuating and application braking torque, appropriately

$\mathrm{TF}^{*}, \mathrm{TF}$ friction fraction of actuating and application braking torque, appropriately

$\mathrm{vV} \quad$ longitudinal velocity of the vehicle

vw longitudinal velocity of the wheel

$\omega \quad$ angular speed of the wheel

\section{Introduction}

Though a specific performance of energy converters is getting better with each passing year, the overall efficiency of transportation has dropped from $26 \%$ to $21 \%$ during the past 70 years due to rising car ownership, heavier vehicle power, much more frequent trips, and longer travelled distances [1]. At this, 20 to $70 \%$ of vehicle energy is lost during braking [2], [3]. Therefore, the problem of energy recovery in braking is currently of great importance. In the case of gradual braking at downhill driving, deceleration ahead traffic lights, parking, and similar regimes where the driver reduces car velocity in a stable and smooth manner, many useful solutions for green energy electric vehicles (EV) can be found in literature [4], [5]. On the other side, very little researches are published about energy saving in intensive antilock braking systems (ABS), such as [6]. Even less attention is paid to energy recycling solutions that might be so universal that equally succeed in both braking scenarios [7].

The benefits of EVs are based on their advantages in terms of the braking management potential, such as fast and accurate power generation by electrical motors, easy torque measurement by sensing the current, accurate wheel speed encoding, and an ability to adjust independently each wheel due to the small motor dimensions [8]. However, in most of existing EV control strategies, regenerative electrical braking (EB) accounts for only a small fraction of the total braking force, which causes high braking security along with poor braking 
economics [9]. The challenge lies in the contradiction between the specific energy requirements posed by intensive stopping and by stopping with energy recycling. In ABS, the friction (hydraulic) brakes (FB) are mostly used that are capable to convert friction force to heat very fast. At gradual braking, EB is preferred in terms of energy recovery, which, however, is restricted in the pace due to the limited state of charge (SOC), voltage, and current. To resolve this problem, the blended braking systems are designed that unite traditional FB and EB, being associated with hybrid energy storage (HES) equipment composed of batteries on one side and ultracapacitors or/and flywheels on the other [10].

With both EB and FB, the braking strength is to be additionally limited to avoid skidding due to tire-road friction weakening. At present, there are no affordable sensors to identify nonlinear and nonstationary tire-road friction and make these data available to a braking controller [11]. Although many attempts have been made, an accurate and general mathematical model of tyre behaviour has not been obtained. Therefore, some indirect estimates are needed that look problematic in general and complicated by the fact that tire properties depend on variable road conditions and many other features.

In contrast to friction, wheel slip can be calculated easily as follows:

$$
\lambda=\frac{v-\omega r}{v}
$$

where $v$ - longitudinal vehicle velocity, $\omega-$ angular wheel speed, and $r$-effective radius of the wheel. EVs usually utilise wheel-based sensors to measure the angular speed and/or angular acceleration of the wheel. As well, a number of different techniques have been proposed to estimate vehicle velocity [3], [12].

To transfer from slip to friction, several methods are available in the literature, such as Pacejka's "Magic Formula", Burckhardt model, Rill model, and others [8], [13], [14]. However, there exist diverse views on how to apply the obtained friction-slip estimates to ensure both the requested stopping rate and maximal energy recovery taking into account that the friction peak optimality for a given road surface is variable. For example, in [7] the highest friction is developed at the slip level of about $12 \%$ on the dry road and at $5 \%$ on the icy road. On the contrary, in [12] the biggest friction appears at $20 \%$ slip on the dry road and at $30 \%$ on the icy road. Usually, the location of these peaks depends on the initial car velocity and alternates for forward-rear and right-left wheels. Hence, to avoid skidding, the designers commonly offer to choose some understated slip level, say $10 \%$ [12]. As a result, the vehicle does not decelerate fast enough and does not save as much energy as desired.

To exclude the above vagueness, in [7] the torque gradient control method is designed, where the derivative $\frac{d T}{d \lambda}$ of the application torque $T$ with respect to slip $\lambda$ is used as a control feedback instead of slip applied in conventional intelligent braking ABS, such as [12]. The main benefit of this method is that it does not require any tire model for implementation. Therefore, this approach is further elaborated in this paper.

Different controllers are applied to manage braking in EVs with blended braking systems. Among them, the intelligent control equipment, including fussy logic, model predictive, genetic algorithm, or neural network (NN) based controllers (NNC), have proven to be an emerging and effective solution capable to deal successfully with the nonlinear, uncertain, and varying braking dynamics [12]. The scope of application of intelligent vehicles with highly nonlinear dynamics driven upon the changeable road pavement grows annually [10].

The NNCs can outperform human experts used in fuzzy logic and may be realized within reasonable timeframes more accurately than other controllers [15]. For these reasons, different $\mathrm{NN}$ methodologies have been applied in the braking systems of automobiles [16], including control in changing driving scenarios [17], uncertain variable-pavement roads [18], and such surface types and combinations as gravel wet, gravel dry, cobblestone wet, cobblestone dry, asphalt wet, and asphalt [12]. The study [19] represents an example of an intelligent system where the braking space is defined as a set of actions covering no braking, weak braking, average braking, and strong braking, which management policy is conducted by deep reinforcement learning.

The NNCs are employed successfully for optimisation of HES energy recovery. For instance, in [20] a convolutional NN estimates EV energy consumption. In [21], a regenerative EB scheme is offered to transfer braking energy to the HES devices. To that end, the multilayer feedforward $\mathrm{NN}$ provides satisfactory capability comprising EV speed and SOC of the supercapacitor and battery banks in a number of braking situations. As well, in [22], consumed power, trip time, and SOC are used as the NN training inputs; the EV recommended operation modes appear on the NN output; whereas the peak and off-peak load times, human behaviour, seasonal and weather conditions feed the model to generate a realistic load pattern. Another example is a deep NN-based approach of EV energy demand estimation proposed in [21], which is based on a driving cycle series served as a static input to the NN. In [24], the HES-based braking system implements automatic control of the EV regeneration torque aiming to improve energy efficiency along with driver's comfort. To apply this system, the accurate prediction of the vehicle deceleration states was produced using the longshort-term memory and a two-layer conventional NN model.

An objective of the current research is to offer a new NNbased control system, which ensures high quality blended braking of EVs in both the intensive (ABS-fed) and the gradual braking scenarios with energy recovery at the changing road pavement. The main contribution of the paper is in the design of the NNC capable to meet the conflicting requirements of different braking modes and road surfaces. This is one of the first studies where the NNC provides the torque gradient control without a tire model resulting in returning maximal energy to the vehicle HES during the braking process. 
Using the author's torque allocation algorithm, the system determines how to distribute the driver's single torque request into separate torque requests between the $\mathrm{FB}$ and EB. Simulation demonstrates effectiveness of the proposed NN-based braking system. The model states and inputs are employed here as a guidance to develop a coupled twolayer NN. Furthermore, the simulation study shows that the designed model can capture various dynamic behaviours that could not be included in the simplified physics-based model. An experimental part of the research proves the model and simulation validity.

In the next sections, an NN-based braking system and its parts are introduced. Then, two design phases of the NNC are explained, which are identification of the $\mathrm{NN}$ model for predicting the $\mathrm{EV}$ behaviour and training the $\mathrm{NNC}$ using the identified NN model. After the control system is trained and validated, its responses are estimated against different driver's requests and environmental conditions. To verify the effect of the proposed control strategy in the hardware environment, an experimentation is described. Finally, results are discussed and conclusions are drawn.

\section{Braking System}

The braking system discussed in this paper involves the reference system (REF), the NN-based model reference controller (MRC), the torque allocation (TA) module coupled with the physics-based EV model (PBEV) with blended braking supplied from the hybrid energy storage (HES), and the computational modules that estimate wheel slip and torque gradient (Fig. 1).

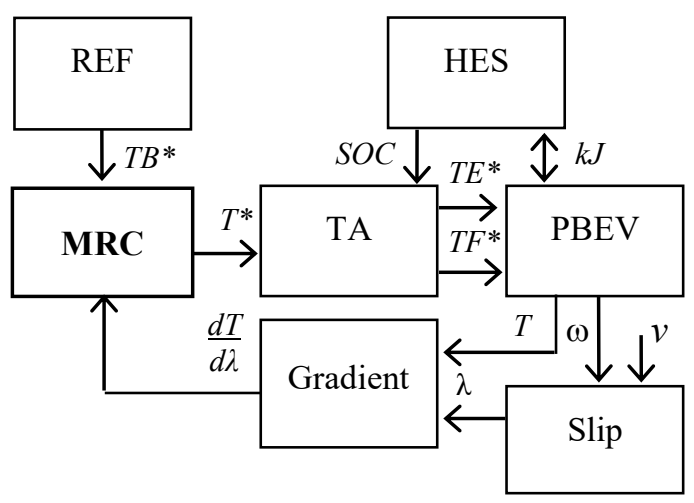

Fig. 1. The architecture of the EV braking system.

The block REF imitates an instant when a driver has stepped on the brake pedal. The block MRC produces actuating torque $T^{*}$ further divided by the block TA into two fractions: electrical $T E^{*}$ and friction $T F^{*}$, both coming to the block PBEV. During braking, the block HES consumes an electrical fraction $k J$ of energy generated by PBEV while its value does not exceed the permissible $S O C$, voltage, and current restrictions. Application torque $T$ of PBEV decelerates the EV with such intensity as to meet the driver's reference $T B^{*}$ on the one hand and to avoid wheel skidding coursed by the wheel slip on the other. Electrical current recharges the HES block from the $\mathrm{EB}$ while the pressure signal adjusts the FB.

The physics-based vehicle model PBEV was established in [7] based on experimental data that describe the interacted single-wheel rotational properties and the EV physical features. It is made up of an adjustable electrical drive implementing regenerative $\mathrm{EB}$, a friction drive integrated with FB, and a module simulating the gear and vehicle inertia. To ensure the direct torque control with consideration of system non-linearity and space vector modulation, the AC6 Interior PM Synchronous Motor Drive block from the Specialized Power Systems MATLAB/Simulink ${ }^{\mathrm{TM}}$ library is used as a core of the EB. It is composed of the electric motor of $288 \mathrm{VDC}, 100$ $\mathrm{kW}$, the three-phase voltage source inverter fed by direct voltage from the HES, the space vector modulated controller equipped with a three-phase current regulator, and the speed controller operated in the torque regulation mode.

The torque allocation module TA algorithmically distributes actuating braking torque $T^{*}$ generated on the $\mathrm{MRC}$ output between the front and rear wheels in a fixed ratio [25] and allocates it between FB and EB based on real-time $S O C$, voltage, and permissible EB current. In [7], an appropriate torque allocation algorithm is offered. In order to keep the HES battery and ultracapacitor within their safe operating areas, this algorithm checks if electrical current and actuating motor torque meet the real-time HES restrictions. Once the braking torque exceeds these boundaries, the FB is added or is replaced with the EB. Therefore, the common trait of this strategy is to include regeneration into all braking scenarios, even during heavy braking with $\mathrm{ABS}$, leaving the solo FB only if the HES is saturated.

\section{Model Reference Controller}

To design the NN, the Deep Learning Toolbox ${ }^{\mathrm{TM}}$ from MATLAB/Simulink ${ }^{\circledR}$ was used in this study. An interactive environment for developing the $\mathrm{NN}$ controllers, namely, the MRC block, was applied. The MRC solves the problem of effective EV braking with maximal recovery of braking energy using data generated by the PBEV. For that, in response to the reference in the form of the driver's torque request $T B^{*}$, the MRC has to produce the actuating braking torque command $T^{*}$ for stopping the PBEV without wheels locking at a variety of road conditions and $\mathrm{EV}$ speeds.

The MRC includes two NNs, which are the NN controller (NNC) generating the actuating braking torque command and the NN model of the PBEV (NNEV) predicting its behaviour. Two phases of the MRC design were conducted (Fig. 2), namely, (a) identification of the NNEV and (b) training the NNC based on the identified NNEV.

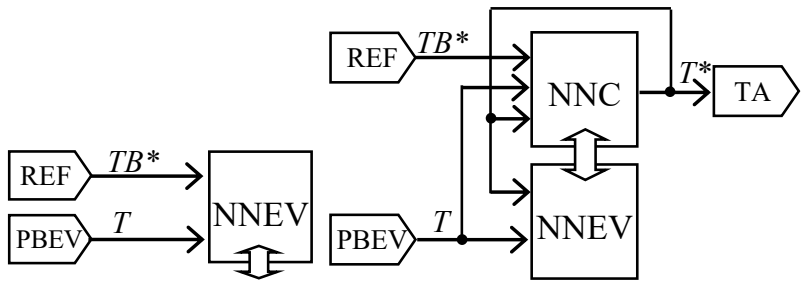

a.

b.

Fig. 2. Two phases of the MRC design: (a) identification of the NNEV, (b) training the NNC. 
The goal of the NNEV identification is to obtain the NNEV parameters with the capacity of representing the behaviour of the unknown PBEV.

The mean squared error (MSE) between the PBEV and NNEV outputs was used as the NNEV training signal. In the NNEV, the feedforward topology is used, and in the $\mathrm{NNC}$ - the recurrent one. In both NNs, a double-layer architecture is applied with delay lines that hold the previous values of braking torque in such a way that the input signals enter the input layers of the NNs in the next cycle.

A variant of the Levenberg-Marquardt gradient descent algorithm [26] was employed for identification and training. Preliminary, a set of samples was prepared that cover the range of expected MRC inputs. The reference training function trainlm is utilised consisted of a series of steps of random heights and random intervals. Since the MRC output is limited to 0 and 1, the sample datasets have been exposed to preprocessing. Both NNs were trained for function approximation (nonlinear regression) so that the MRC output follows the reference as closely as possible. Some sample characteristics of the training process are collected in Table I.

Table I. Characteristics of training samples

\begin{tabular}{|l|c|c|}
\hline \multicolumn{1}{|c|}{ Sample parameter } & Unit & Value \\
\hline Sampling interval & $\mathrm{s}$ & 0.05 \\
\hline Number of samples & & 400 \\
\hline Maximum reference interval & $\mathrm{s}$ & 2 \\
\hline Minimum reference interval & $\mathrm{s}$ & 0.1 \\
\hline
\end{tabular}

The REF block was adopted as a source to generate training data. In the NNEV identification phase, the batch mode was enforced in which all inputs in the training dataset were applied to the NNEV before the weight updating. It was performed offline based on the measurements of the NNEV inputs and outputs of the system. The NNEV output was compared to the PBEV output aiming to minimise the MSE between the responses. During identification, the sample dataset was shared among the training, validation, and testing phases.

After performing identification, a final NNEV architecture was chosen, which has two neurons in the input layer, 10 neurons in the only hidden layer with the sigmoid activation functions, and an output layer with a single neural linear activation function. The sigmoid tansig activation functions allow the NNEV to learn nonlinear relationships between inputs and output. The linear purelin activation function is suitable for solving the regression problem related to the braking torque estimation. It was found that adding more hidden layers or hidden neurons does not improve the performance while increasing computation complexity, which is not desirable in a time critical context. Another obstacle to increasing the number of hidden layers might be the opacity of the system, which prevents effective security checks. The convergence curve of the model shows that MSE has almost converged at the 10 th epoch where its value is $7.6574 \mathrm{e}^{-06}$. Therefore, the model can be qualified as a well-trained one and 10 epochs chosen for training are sufficient to meet the demands of the model application. The final regression value of 0.99997 after training, validation, and testing, also qualifies the model as a good one.
Once the NNEV is identified, it was used to train the NNC. Among the algorithms suitable for the NNC performance optimising, the fastest of the gradient types was applied, namely, the backpropagation algorithm able to perform computations backward through the NN. The NNC was trained using the PBEV model, having one input and one output to generate data for the MRC training algorithm. The strategy of training supports both the offline and online tuning the $\mathrm{NNC}$ weights in a way that the MSE between the reference and the NNC output converges to almost zero.

The chosen NNC architecture has 3 neurons in the input layer, 10 neurons in the only hidden layer of the sigmoid activation function, and an output layer with a singleneural linear purelin activation function. However, during the $\mathrm{EV}$ operation, the NNC can continue to learn and adjust the hidden layer parameters in accordance with changing operating conditions. As follows from the training results, the convergence speed of the $\mathrm{NNC}$ is very fast, and the optimization is obtained quickly. According to the convergence curve, the MSE has almost converged at the 10th epoch, where the value is $108.35 \mathrm{e}-06$. A regression value of 0.99933 qualifies the $\mathrm{NNC}$ model as well trained.

\section{Results and Discussion}

After the MRC is trained and validated, the PBEV model responses were estimated against different driver's requests and MRC commands. To verify the effect of the designed control strategy in the hardware environment, a set of hardware-in-loop (HIL) experiments was preliminary conducted. The experimentally validated HIL emulated an electric sport utility vehicle with four independent in-wheel motor powertrains in dynamically changing and vaguely defined environmental conditions [6]. The core of the HIL setup is represented by a decoupled FB testbed developed by the ZF TRW Automotive $^{\circledR}$ (Koblenz, Germany), connected to the high-precision vehicle dynamic software IPG CarMaker ${ }^{\circledR}$ (Karlsruhe, Germany). The mass of the sport utility vehicle under the study is $2117 \mathrm{~kg}$ and wheel radius is 0.2 $\mathrm{m}$. It was assumed that the vehicle is moving in a straight-line manoeuvre at a velocity of $100 \mathrm{~km} / \mathrm{h}$, powered by an electrical motor with a maximal permissible torque of $200 \mathrm{Nm}$, wheel speed of $157 \mathrm{rad} / \mathrm{s}$, and inertia of $2.1 \mathrm{kgm}^{2}$, connected to the wheel imitator through the gear with a ratio of 10.5 . The HIL system under investigation provides separate control of each of the four wheels via two fundamentally distinct drives. An electrohydraulic brake of the HIL setup was connected to a host computer through the $\mathrm{dSPACE}^{\circledR}$ electronic platform. The IPG CarMaker ${ }^{\circledR}$ software was running by the EV model.

In Fig. 3, several experimental results obtained from the HIL at braking on the changing road surface from dry to icy are shown. The wheel velocity curve (vw) demonstrates the velocity of the front left wheel, which follows the vehicle longitudinal velocity (vv). Its wheel slip (L\%), friction torque (TF), and electrical torque (TE) curves are displayed. On the dry asphalt, since the EB 
torque is not sufficient to retain the optimal slip, the control system requests additional FB torque. As the speed drops to about $50 \mathrm{~km} / \mathrm{h}$, the road under the $\mathrm{EV}$ wheels changes from dry to icy. On an icy road, the FB is no longer required, as the electrical torque is sufficient to slow the car down within the optimal wheel slip zone. Therefore, regeneration lasts all the way, while the FB does not participate in braking, except for the end of the process. At the end of the slowing down, the regeneration is turned off, and FB completes the deceleration alone. An evident chattering phenomenon at low velocity is seen in the experimental torque plots.

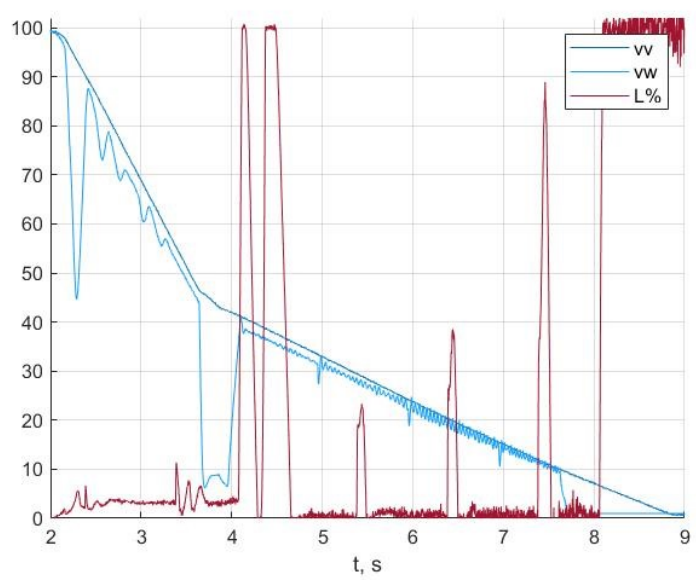

a.

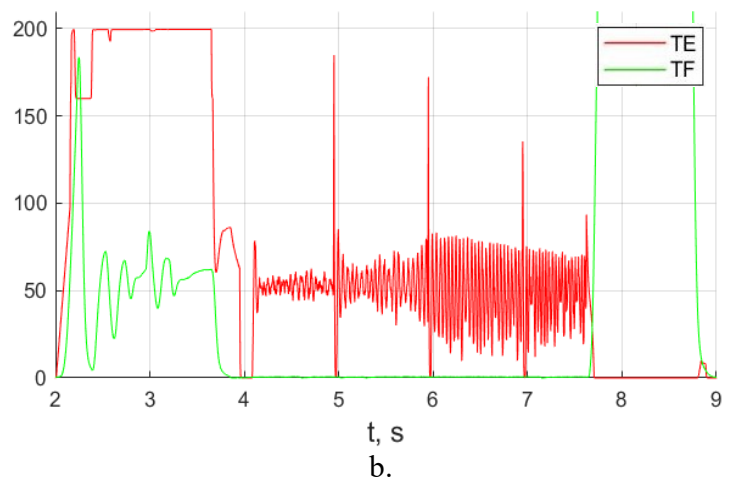

Fig. 3. Traces obtained from experimentation: a - vehicle (blue) and wheel (light blue) velocities and wheel slip (purple); b regenerative (red) and friction (green) torque.

In order to investigate the effect of the NN control, taking into account the assessment of the road surface, the motion of a vehicle on a changing pavement, from dry to icy, was simulated. Figure 4 displays the traces obtained from the simulation of the volatile driving conditions. Here, in response to a driver's setpoint $T B^{*}$, the application torque was allocated between the electrical $\left(T E^{*}\right)$ and friction $\left(T F^{*}\right)$ fractions, the first of which was limited by the value of $200 \mathrm{Nm}$.

As follows from Fig. 4, the system successfully detects a change of the road surface based on analysis of the torque gradient. At the beginning, the deceleration was around $30 \mathrm{~m} / \mathrm{s}^{2}$ on a dry surface. Since the road surface changes from dry to icy, the new gradient is recognized, and the total application torque needed to ensure an intensive stop drops to $60 \mathrm{Nm}$. The FB is no longer required, as the electrical torque is sufficient to slow the car down within the optimal wheel slip zone. Therefore, only electrical braking is performed further. At low velocity, the EB turns off, and the FB runs alone. Herewith, an obvious reduction in chattering is observed in the torque plots at low velocity. First, this is because the designed model takes into account an increase of friction due to its static fraction. Second, because the torque loop of the electrical drive remains closed, even without the EB.
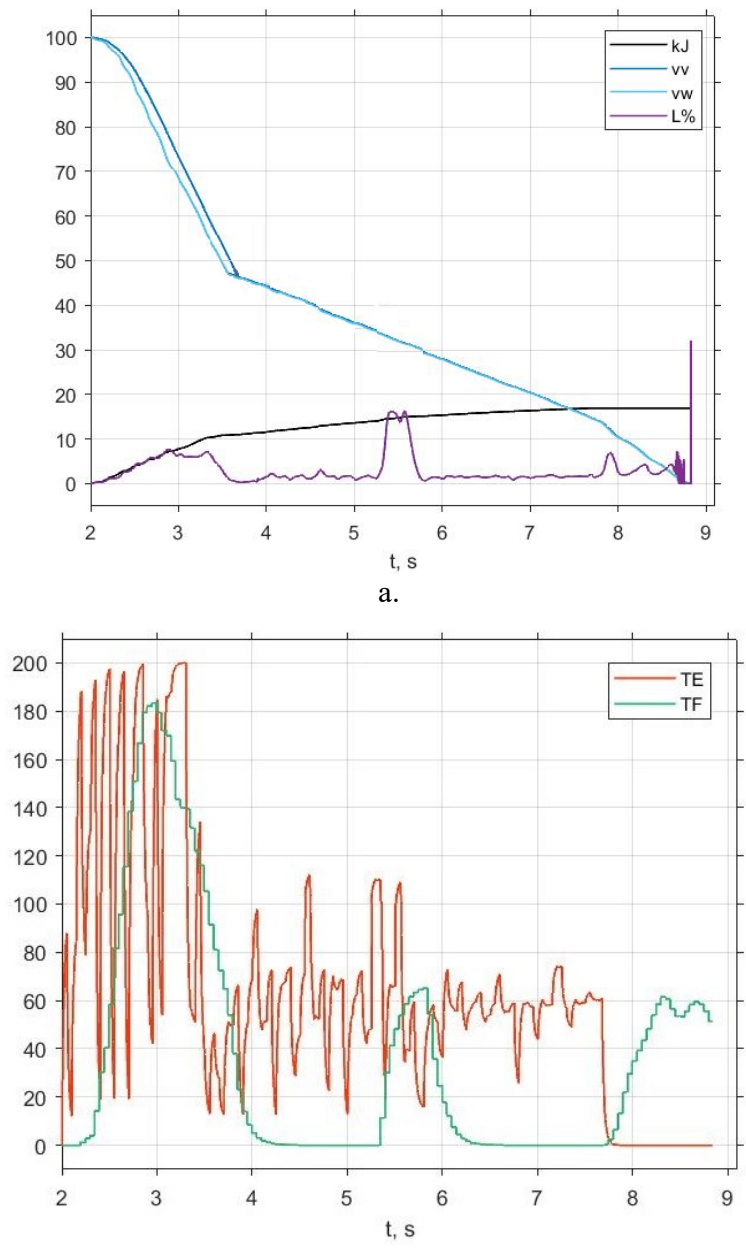

b.

Fig. 4. Traces obtained from simulation: a - vehicle (blue) and wheel (light blue) velocities and wheel slip (purple); b regenerative (red) and friction (green) torque.

Based on the energy curves (kJ, black) and assuming a $50 \%$ regenerative efficiency, it turns out from Fig. 4 that nearly $22 \mathrm{~kJ}$ of energy is recovered when braking on all roads.

\section{Conclusion}

An NN-based control system offered in this paper ensures high quality blended braking of the green energy vehicles in all deceleration scenarios, with energy recovery at the changing road pavement.

By imitating changes in road conditions, the robustness of the designed MRC was evaluated. Comparison of the experimental and simulation results on two different road surfaces has proved good braking performance established with MRC. When trained on a combination of data from different roads, the NN control model is able to make appropriate predictions for the road surface on which the EV is traveling. 
The EV velocity, slip, and torque responses have proved the proper system performance. The MRC-driven system supports the same torque as in experimentation, successfully keeps optimal wheel slip in braking, and demonstrates the same braking times as the experimental system shows. At the same time, the torque chattering is minimal upon the NN control.

An ability to execute accurately indicates that the model deals properly with the strong nonlinearity and cross coupling of the EV dynamics. At the same time, this system also has the characteristics of flexible control and prospective applicability.

\section{Acknowledgement}

This work was supported by the Estonian Research Council grant PRG 658.

\section{References}

[1] V. Smil, "Increasing energy inefficiency", IEEE Spectrum, 2 (2021). https://spectrum.ieee.org/energy/policy/increasingenergy-inefficiency (assessed on 12.03.2021).

[2] M. Shang, L. Chu, J. Guo and Y. Fang, "Hydraulic braking force compensation control for hybrid electric vehicles," International Conference on Computer, Mechatronics, Control and Electronic Engineering (CMCE), Changchun, China, pp. $335-339$ (2010).

[3] S. M. Savaresi and M. Tanelli, Active Braking Control Systems Design for Vehicles, London: Springer, $200 \mathrm{p}$. (2010).

[4] Z. Raud and V. Vodovozov, "Reserves for regenerative braking of battery electric vehicles," Electric Power Quality and Supply Reliability Conference (PQ), Rakvere, Estonia, pp. 1 - 6 (2014).

[5] V. Vodovozov and T. Lehtla, "Design considerations for propulsion drives of electric vehicles," 14th International Symposium "Topical problems in the field of electrical and power engineering. Doctoral school of energy and geotechnology II," Pärnu, Estonia, pp. 35 - 39 (2014).

[6] A. Aksjonov, V. Vodovozov, K. Augsburg and E. Petlenkov, "Design of regenerative anti-lock braking system controller for 4-in-wheel-motor drive electric vehicle with road surface estimation," Int. J. Automotive Technology, 19(4), pp. $727-742$ (2018).

[7] V. Vodovozov and Z. Raud, "Modelling of energy recovery in electric vehicles for various braking scenarios on changing road surfaces," Renewable Energy and Power Quality Journal, 18, pp. 178 - 183 (2020).

[8] M. Cecotti, J. Larminie and B. Azzopardi, "Estimation of slip ratio and road characteristics by adding perturbation to the input torque," IEEE International Conference on Vehicular Electronics and Safety (ICVES), Istanbul, Turkey, pp. $31-36$ (2012).

[9] H. Hea, C. Wanga, H. Jiaa and X. Cuic, "An intelligent braking system composed single-pedal and multi-objective optimization neural network braking control strategies for electric vehicle," Applied Energy, 259 (2020).

[10] F. Naseri, E. Farjah and T. Ghanbari, "An efficient regenerative braking system based on battery/ supercapacitor for electric, hybrid, and plug-in hybrid electric vehicles with BLDC motor," IEEE Transactions on Vehicular Technology 66(5), 3724 - 3738 (2017).
[11] W. Hong, I. Chakraborty and H. Wang, "Parametric optimization problem formulation for connected hybrid electric vehicles using neural network based equivalent model," IEEE 90th Vehicular Technology Conference (VTC), Honolulu, HI, USA (2019).

[12] K. M. Sanjay and C.-C. Lim, "A neural network based anti-skid brake system," Discrete and Continuous Dynamical Systems, 5(2), pp. 321 - 338 (1999).

[13] B. Wang, H. Guan, P. Lu and A. Zhang, "Road surface condition identification approach based on road characteristic value," J. Terramech, 56, pp. 103 - 117 (2014).

[14] R. Bhandari, S. Patil and R. K. Singh, "Surface prediction and control algorithms for anti-lock brake system," Transp. Res. Part C, 21, pp. 181 - 195 (2012).

[15] X. An, W. Zhu and N. An, "Control system optimization of spillage brake based on RBF neural network," Concurrency and Computational Practice and Experience, $32(2020)$

[16] O. O. Bamigboye and S. E. Obaje, "Intelligent automatic car braking control system using neural network classifier," International Journal of Engineering Inventions, 5(06), pp. 51 - 56 (2016).

[17] S. Kuutti, R. Bowden, H. Joshi, R. de Temple and S. Fallah, "Safe deep neural network-driven autonomous vehicles using software safety cages," 20th International Conference on Intelligent Data Engineering and Automated Learning (IDEAL), Manchester, UK, Part II, pp. 1 - 11 (2019).

[18] H. Mirzaeinejad, "Robust predictive control of wheel slip in antilock braking systems based on radial basis function neural network," Applied Soft Computing, 70, pp. $318-$ 329 (2018).

[19] H. Chae, C. M. Kang, B. D. Kim, J. Kim, C. C. Chung and J. Wю Choi, "Autonomous braking system via deep reinforcement learning," IEEE 20th International Conference on Intelligent Transportation Systems (ITSC), Yokohama, Kanagawa, Japan, pp. 1 - 6 (2017).

[20] S. Modia, J. Bhattacharyaa and P. Basak, "Estimation of energy consumption of electric vehicles using deep convolutional neural network to reduce driver's range anxiety," ISA Transactions, 98, pp. $454-470$ (2020).

[21] K. Kiddee, "Hybrid energy harvesting system based on regenerative braking system and suspension energy harvesting for middle electric vehicle," TNI Journal of Engineering and Technology, 8(2), pp. 29 - 38 (2020).

[22] S. Morsalin, K. Mahmud and G. Town, "Electric vehicle charge scheduling using an artificial neural network," IEEE Innovative Smart Grid Technologies - Asia (ISGTAsia), Melbourne, Australia, pp. 276 - 280 (2016).

[23] J. Topi'c, B. Škugor and J. Deur, "Neural network-based modeling of electric vehicle energy demand and all electric range," Energies, 12, 1396 (2019).

[24] K. Min, K. Yeon, Y. Jo, G. Sim, M. Sunwoo and M. Han, "Vehicle deceleration prediction based on deep neural network at braking conditions," International Journal of Automotive Technology, 21(1), pp. 91 - 102 (2020).

[25] Y. Tao, X. Xie, H. Zhao, W. Xu and H. Chen, "A regenerative braking system for electric vehicle with four in-wheel motors based on fuzzy control," 36th Chinese Control Conference, Dalian, China, pp. 4288 - 4293 (2017).

[26] H. P. Gavin, The Levenberg-Marquardt Algorithm for Nonlinear Least Squares Curve-Fitting Problems, Durham: NC, Duke University, 19 p. (2020). 